\title{
The metabolic pathways, metabolites and signal transduction
}

\author{
C.K. Mitra \\ Department of Biochemistry, University of Hyderabad, 500046 Hyderabad, India \\ e-mail:ck.mitra@gmail.com
}

Key words: dynamics, metabolites, pathways, enzyme kinetics, quasi-steady state

Motivation and Aim: Although the metabolic pathways have been extensively studied we only have a static picture of the complete system. It is also true that we know reasonably well the acceptable concentration limits (physiologically meaning) of a large number of metabolites, but we do not know the dynamics, i.e., the fluxes (including sources and sinks) of most of the important metabolites. For a living system, the fluxes are more relevant compared to their static concentrations. Most of the metabolite concentrations must stay within a well-defined limit (safe volume in the multidimensional hyperspace described by the metabolite concentrations).

All metabolic fluxes are products of biochemical reactions that are catalyzed by enzymes. Practically all biochemical reactions are catalyzed by enzymes and each enzyme is characterized by at least two parameters determined by the substrate. The two most common parameters, $\mathrm{V}_{\max }$ and $\mathrm{K}_{\mathrm{m}}$, are usually defined in terms of the Michealis-Menten rate equation (single substrate, single intermediate enzyme kinetics). For multiple substrate reactions, additional $\mathrm{K}_{\mathrm{m}}$ must be defined. For many enzymes, there are regulatory sites and additional information on the binding affinity of the regulatory ligand need to be known. Therefore it is not uncommon to describe an enzyme by a set of six or more parameters [1].

Methods and Algorithms: We use an approximate method that we have earlier developed for the study of reaction kinetics [2]. In brief, we express the substrate concentration in units of the $\mathrm{K}_{\mathrm{m}}$, therefore reducing the substrate concentration to a dimensionless number. In all enzyme catalyzed reactions, we observe a limiting rate (rate reaching a maximum value) with increase in the substrate concentration and we therefore replace $[\mathrm{S}]$ with $[\mathrm{S}] /(1+[\mathrm{S}])$ in the kinetic equations. Other allowances are made to conform known biochemical properties. Regulators (inhibitory) are modeled as $1 /(1+[\mathrm{I}])$ where I the inhibitor concentration expressed in terms of $\mathrm{K}_{\mathrm{I}}$ where $\mathrm{K}_{\mathrm{I}}$ is the dissociation constant for the enzyme-inhibitor complex. Finally the set of first order differential equations were set up and solved using Octave [3].

Results: The results obtained from the software are presented graphically. As an example, we have simulated the common symport and antiport (ion transporters) widely found on membrane surfaces that preserve charge balance.

Conclusion: The results would have been more interesting if we could include the membrane potential also in the overall fluxes.

\section{References}

1. Enzyme Database - BRENDA https://www.brenda-enzymes.org/; see also: Schomburg I., Chang A., Schomburg D. (2002) BRENDA, enzyme data and metabolic information. Nucleic Acids Research. 30(1):47-49.

2. Korla K., Mitra C.K. (2014) Kinetic modelling of coupled transport across biological membranes. Indian Journal Biochem Biophys. 51(2):93-99.

3. GNU Octave: A scientific programming language. https://www.gnu.org/software/octave/; version used in the current work is 4.4.0. 